\title{
Reticulação do colágeno corneano com radiação ultravioleta e riboflavina para tratamento do ceratocone: resultados preliminares de um estudo brasileiro
}

\author{
Corneal collagen crosslinking with riboflavin and \\ ultraviolet radiation for keratoconus treatment: \\ preliminary results of a Brazilian study.
}

Ricardo Lamy ${ }^{1}$, Camila Fonseca Netto², Mariana Gomes Pecego ${ }^{3}$, José Guilherme de Carvalho Pecego ${ }^{4}$, Basílio de Bragança Pereira ${ }^{5}$, Haroldo Vieira de Moraes Junior ${ }^{6}$, Adalmir Morterá Dantas

\section{RESUMO}

Objetivo: Avaliar o efeito clínico e topográfico da reticulação do colágeno corneano (crosslinking) em pacientes com ceratocone pertencentes à população brasileira. Métodos: Trinta e sete olhos de 37 pacientes (22 mulheres e 15 homens) com diagnóstico de ceratocone em progressão e com espessura mínima de 395 micra, foram submetidos à técnica de indução da reticulação do colágeno corneano através da aplicação durante 30 minutos de luz ultravioleta $(370 \mathrm{~nm})$ associada à solução de riboflavina $0,1 \%$ (instilada a cada 5 minutos). Foram comparados os valores ceratométricos e a melhor acuidade visual corrigida antes e após o procedimento, com intervalos de 40 e 90 dias. Resultados: Houve redução do poder dióptrico médio (PDM) nos $3 \mathrm{~mm}$ centrais em $83,8 \%$ dos pacientes após três meses. O PDM reduziu em média $0,4 \mathrm{D}$ nos $3 \mathrm{~mm}$ centrais $(\mathrm{p}<0,01)$ e $0,33 \mathrm{D}$ nos $5 \mathrm{~mm}$ centrais $(\mathrm{p}<0,01)$. O valor ceratométrico máximo reduziu em média $0,6 \mathrm{D}(\mathrm{p}<0,01)$. A acuidade visual corrigida do olho tratado evoluiu com melhora média de 6,27 letras $(\mathrm{p}<0,01)$ após 90 dias. Conclusão: Observamos uma melhora menos acentuada dos índices topográficos e de acuidade visual em comparação aos resultados encontrados em outras populações. São necessários mais estudos com um maior número de pacientes e maior tempo de seguimento para que esta técnica confirme seu propósito de estabilização da doença com apenas uma aplicação, e possa talvez se tornar, no futuro, o método de escolha para o tratamento do ceratocone.

Descritores: Ceratocone/quimioterapia; Ceratocone/metabolismo; Colágeno/efeitos de radiação; Agentes fotossensibilizantes/uso terapêutico; Riboflavina/uso terapêutico; Raios ultravioleta; Topografia da córnea

\footnotetext{
'Pós-graduando nível mestrado em oftalmologia, Médico do serviço de oftalmologia do Hospital Universitário Clementino Fraga Filho da Universidade Federal do Rio de Janeiro - UFRJ, Rio de Janeiro (RJ), Brasil;

${ }_{3}^{2}$ Pós-graduanda nível especialização em oftalmologia da Universidade Federal do Rio de Janeiro - UFRJ, Rio de Janeiro (RJ), Brasil; 'Pós-graduanda nível especialização em oftalmologia da Universidade Federal do Rio de Janeiro - UFRJ, Rio de Janeiro (RJ), Brasil; ${ }^{4}$ Doutor, Médico do serviço de oftalmologia do Hospital Universitário Clementino Fraga Filho da Universidade Federal do Rio de Janeiro - UFRJ, Rio de Janeiro (RJ), Brasil;

${ }^{5}$ Livre-docente, Professor Titular de Bioestatística da Universidade Federal do Rio de Janeiro - UFRJ, Rio de Janeiro (RJ), Brasil;

'Livre-docente, Professor Associado de oftalmologia da Universidade Federal do Rio de Janeiro - UFRJ, Rio de Janeiro (RJ), Brasil; Livre-docente, Professor Titular de oftalmologia da Universidade Federal do Rio de Janeiro - UFRJ, Rio de Janeiro (RJ), Brasil.
}

Trabalho realizado na Universidade Federal do Rio de Janeiro.

Recebido para publicação em: 29/7/2008 - Aceito para publicação em 22/10/2008 


\section{INTRODUÇÃO}

A reticulação polimérica é um processo que ocorre quando cadeias poliméricas lineares ou ramificadas são interligadas por pontes de ligações formando uma rede polimérica tridimensional, processo também conhecido como crosslinking (formação de ligações cruzadas ${ }^{(1-2)}$. A reticulação pode ser de natureza química, formada por ligações covalentes irreversíveis, ou reticulação física, formada por várias ligações reversíveis (ligações iônicas). De uma maneira geral, com o aumento da reticulação, as estruturas tendem a perder sua fluidez e se tornam mais rígidas.

O colágeno é um polímero natural, suas fibras são formadas por fibrilas que por sua vez originam-se de ligações covalentes entre moléculas de tropocolágeno ${ }^{(3)}$. Sabe-se que o processo de envelhecimento da pele humana está intimamente relacionado à reticulação do colágeno ${ }^{(4)}$, principalmente nas áreas mais expostas à luz solar.

Um dos primeiros inventores a encontrar utilidade na indução de ligações cruzadas entre polímeros foi Charles Goodyear, que em 1839, ao derramar acidentalmente sobre uma chapa quente, parte da mistura de látex e enxofre que carregava, observou a formação de uma borracha mais resistente, que chamou de vulcanizada. Considerada uma das maiores descobertas acidentais da história, a borracha vulcanizada é produzida a partir da formação de pontes de ligação entre cadeias de poliisopreno (látex) ${ }^{(5)}$. $\mathrm{Na}$ medicina, Carpentier ${ }^{(6)}$ propôs no final dos anos 60 o uso do glutaraldeído como agente químico capaz de promover a reticulação do colágeno existente nos tecidos utilizados para confecção de próteses valvulares cardíacas. Com esse tratamento foi possível retardar a degradação dos tecidos colágenos e prolongar a vida útil das biopróteses. Na oftalmologia, Harner ${ }^{(7)}$ foi um dos primeiros a provocar a reticulação do colágeno em biomateriais para utilização em epiceratoplastias sintéticas. A reticulação fotoquímica de polímeros teve seu primeiro processo ${ }^{(8)}$ patenteado nos Estados Unidos em 1966. No final da década de 90 , Spoerl, Huhle e Seiler ${ }^{(9)}$ propuseram o uso de luz ultravioleta associada a um agente fotossensibilizante (riboflavina) para indução da reticulação do colágeno corneano (CXL). A técnica demonstrou ser capaz de enrijecer a córnea e apresentouse como uma opção de tratamento conservador para as ectasias corneanas.

O ceratocone é uma distrofia da córnea caracterizada por uma ectasia e consequiente aumento de sua curvatura central, geralmente bilateral e progressiva.
Sua freqüência é de aproximadamente 1:2000 na população, e aproximadamente $20 \%$ dos pacientes podem evoluir com a necessidade de ceratoplastia penetrante. A reduzida reticulação do colágeno corneano em pacientes com ceratocone foi demonstrada por Cannon ${ }^{(10)}$ em 1978. Estudos ${ }^{(11-12)}$ têm demonstrado que a indução da reticulação do colágeno corneano através do uso de radiação ultravioleta A (UVA) associada à riboflavina promove um aumento da rigidez biomecânica da córnea possibilitando a estabilização da doença. Até o presente momento, não há trabalhos publicados, na literatura indexada, envolvendo a população do Brasil. O propósito do presente estudo é avaliar o efeito clínico e topográfico da aplicação de UVA associado à riboflavina em córneas de pacientes com ceratocone pertencentes à população brasileira.

\section{Métodos}

O estudo foi aprovado pelo Comitê de Ética em Pesquisa do Hospital Universitário Clementino Fraga Filho e todos os participantes assinaram um termo de consentimento livre e esclarecido, de acordo com os princípios da Declaração de Helsinque. Foram incluídos no estudo 37 olhos de 37 pacientes (22 mulheres e 15 homens) com ceratocone. A média da idade dos pacientes foi de 24 anos com idade mínima de 18 e máxima de 33 anos. $\mathrm{O}$ diagnóstico de ceratocone foi baseado em critérios topográficos e sinais clínicos tais como afinamento estromal, anel de Fleischer e estrias de Vogt. Usuários de lente de contato e pacientes com opacidades corneanas foram excluídos. A progressão da doença nos 12 meses anteriores ao tratamento foi confirmada através da história clínica e/ou topográfica em todos os pacientes.

Antes do tratamento, todos os pacientes foram avaliados biomicroscopicamente. A obtenção das medidas de acuidade visual sem correção e com correção foi feita com o uso de tabelas ETDRS para teste a quatro metros. A tabela utilizada para refinamento do exame refratométrico era diferente da tabela para o teste da acuidade visual, evitando a memorização dos optotipos. Para determinação da melhor acuidade visual corrigida, utilizamos armações de prova. A iluminação da sala e da tabela foi aferida e controlada semanalmente. A avaliação topográfica da córnea foi realizada através de sistema baseado em discos de plácido e de topografia de varredura em fenda (Orbscan IIzBausch \& Lomb ${ }^{\circledast}$ ). Este exame também permitiu a determinação do ponto corneano mais fino. Foram excluídos os pacientes que apresentaram ponto corneano mais fino inferior a 395 micra em ambos os olhos. Todos os exames foram repetidos com 40 e 90 dias. 


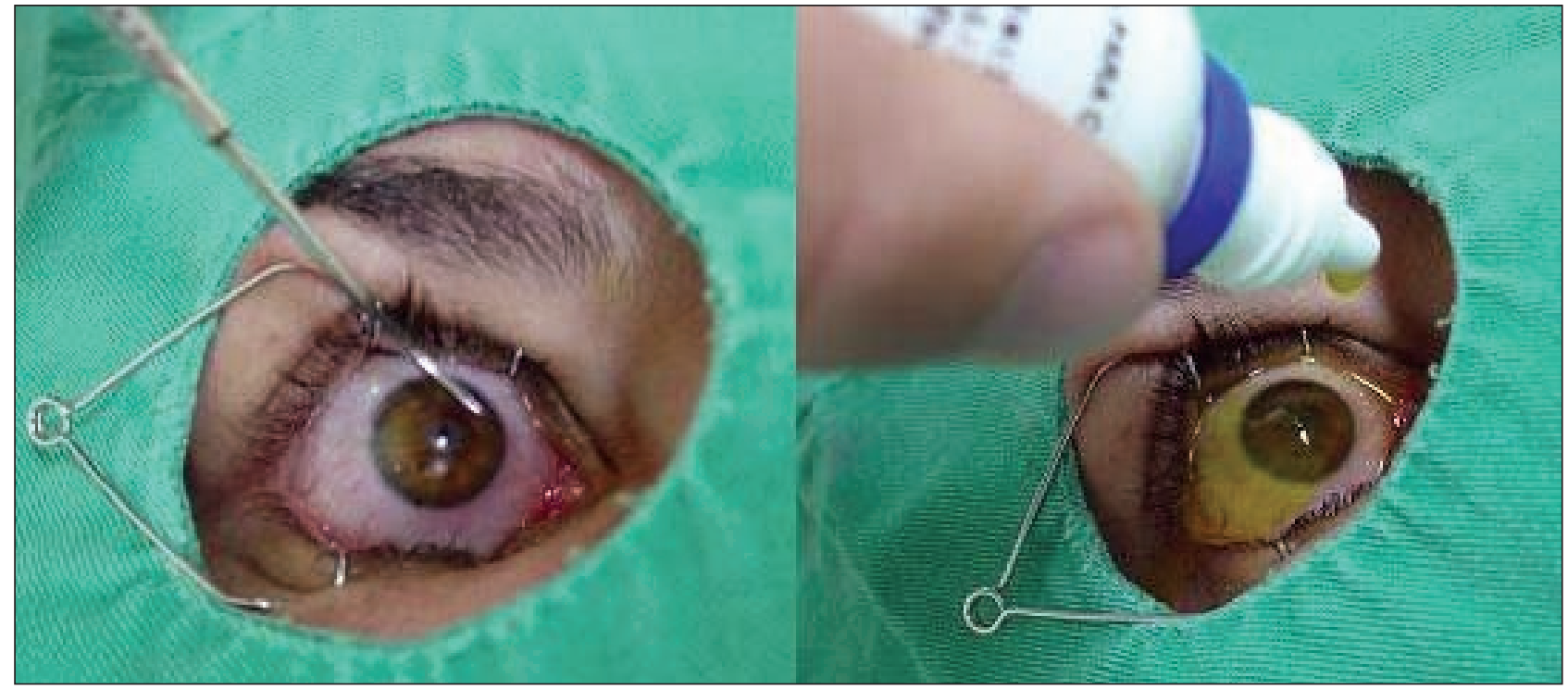

Figura 1: Escarificação dos $8 \mathrm{~mm}$ centrais da córnea seguida pela instilação da riboflavina $0,1 \%\left(\mathrm{Ophthalmos}^{\circledR}\right)$

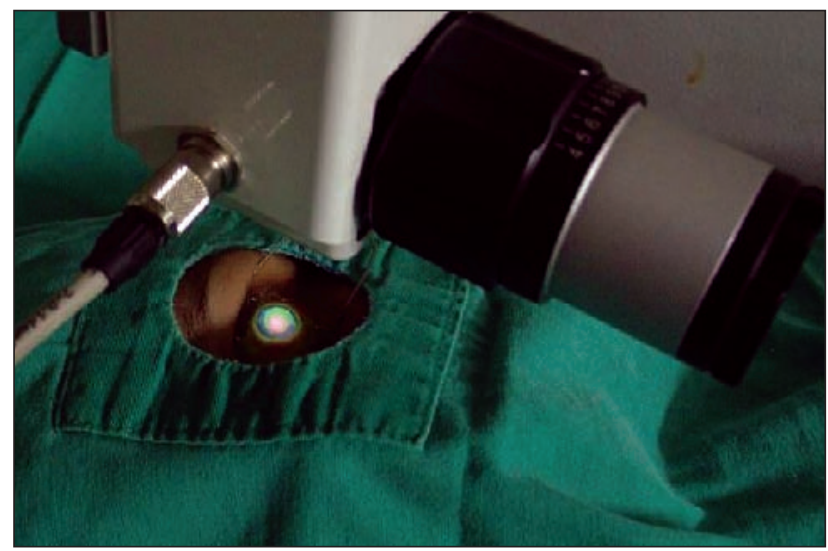

Figura 2: Aplicação de luz ultravioleta (Vega CBM X-linker ${ }^{\circledR}$ ) durante 30 minutos com intervalos a cada 5 minutos para instilação de riboflavina

O olho que apresentou a pior pontuação no teste de acuidade visual corrigida (BCVA) recebeu o tratamento. Cinco pacientes tiveram tratado o olho com a melhor BCVA porque o outro olho apresentava o ponto mais fino da córnea inferior a 395 micra (Orbscan IIzBausch \& Lomb $^{\circledast}$ ).

A técnica para tratamento consiste na aplicação prévia de 1 gota de cetorolaco de trometamina 0,4\% (Acular LS ${ }^{\circledast}$ ) 15, 10 e 5 min antes do procedimento, 1 gota de cloridrato de poximetacaína $0,5 \%$ (Anestalcon $\left.{ }^{\circledR}\right) 10$ e 5 min antes do procedimento, colocação de blefarostato e desepitelização dos $8 \mathrm{~mm}$ centrais da córnea com escarificador, instilação de 3 gotas de riboflavina $0,1 \%$ Ophthalmos $^{\circledast}$ (Figura 1) 12, 8, 4 minutos e imediatamente

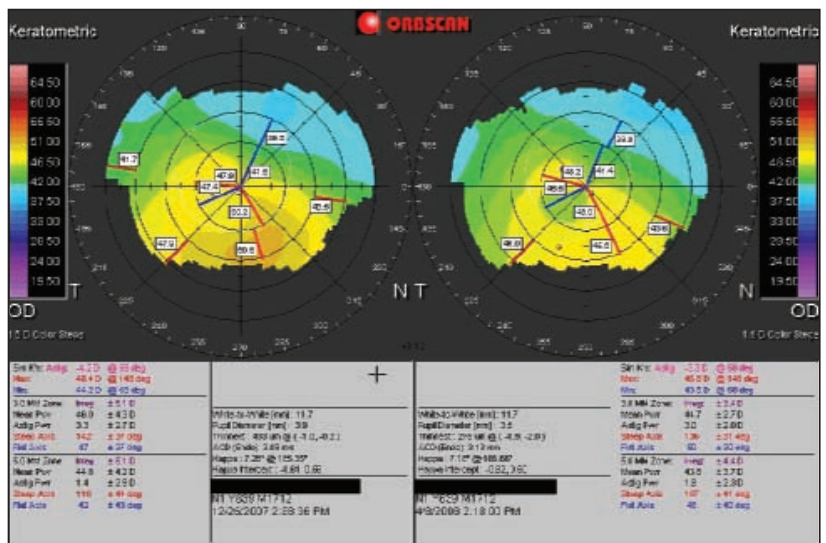

Figura 3: Mapa ceratométrico axial de um mesmo olho, antes do tratamento (à esquerda) e três meses após a indução da reticulação do colágeno (à direita), demonstrando melhora dos índices topográficos e uma redução de $1,3 \mathrm{D}$ no PDM dos $3 \mathrm{~mm}$ centrais

antes de iniciar a aplicação de luz ultravioleta tipo A (UVA) 370nm com irradiância de $3 \mathrm{~mW} / \mathrm{cm}^{2}$ (Vega CBM X-Linker ${ }^{\oplus}$ ). O aparelho é programado para emitir UVA durante 30 min (Figura 2) com intervalos a cada 5 min para instilação de 1 gota da solução de riboflavina. No terceiro intervalo (15 minutos) instilamos mais 1 gota de cloridrato de proximetacaína $0,5 \%$. Após os 30 minutos, a emissão de luz se encerra e o olho é lavado com solução salina $(0,9 \%)$ gelada. Instilamos 1 gota de cetorolaco de trometamina $0,4 \%$ (Acular $\mathrm{LS}^{\oplus}$ ), 1 gota de ofloxacino $0,3 \%\left(\right.$ Oflox $\left.^{\circledR}\right)$ e adaptamos uma lente de contato gelatinosa. Todos os pacientes ingeriram 1 hora antes do procedimento 1 comprimido de lumiracoxibe $400 \mathrm{mg}$ (Prexige ${ }^{\circledR}$ ) e receberam dois comprimidos para uso 


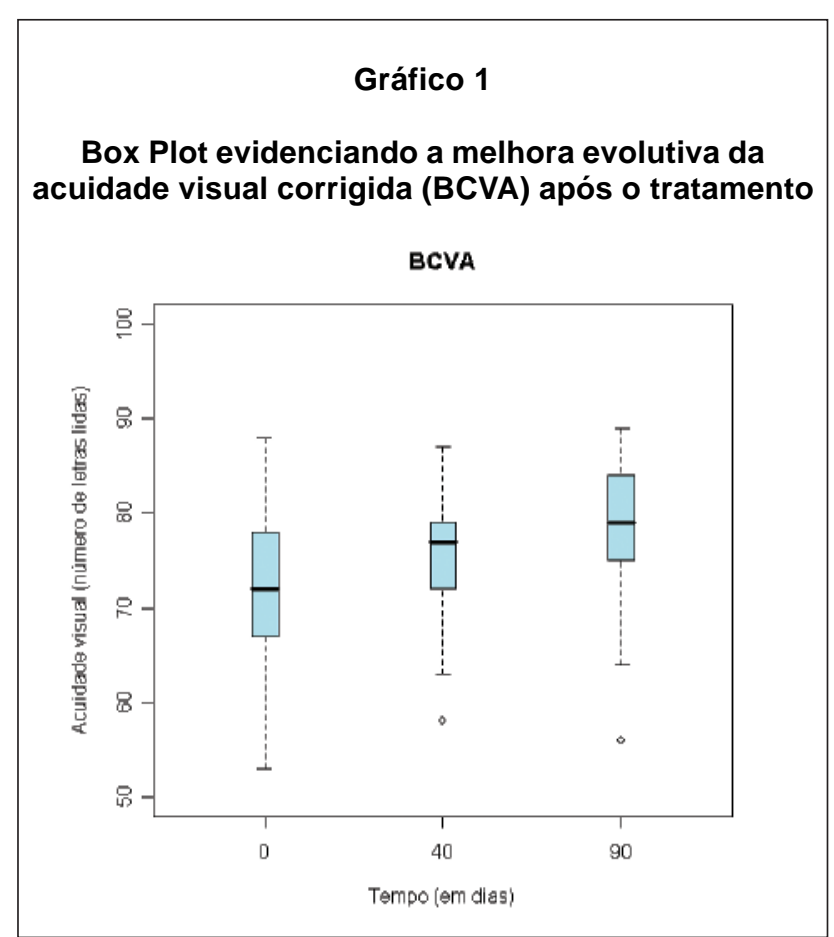

durante 2 dias com posologia de 1 vez ao dia. Receberam ainda amostras de colírios suficientes para manter a seguinte terapia tópica: cetorolaco de trometamina $0,4 \%$ (Acular $\mathrm{LS}^{\oplus}$ ) - $1 \mathrm{gt} 6 / 6 \mathrm{~h}$ por 10 dias; ofloxacino $0,3 \%$ (Oflox $\left.{ }^{\circledR}\right) 1$ gt $6 / 6$ h por 5 dias, Carboximetilcelulose $0,5 \%$ (Fresh Tears ${ }^{\circledR}$ ) uso com freqüência liberada.

As revisões biomicroscópicas foram diárias até que a reepitelização se completasse. Após isso, retiramos a lente e as demais visitas foram programadas para 40 e 90 dias. Os dados obtidos pós-operatoriamente com o Orbscan II $z^{\circledR}$ foram utilizados apenas para avaliação dos parâmetros topográficos da superfície anterior da córnea.

\section{Resultados}

Os dados foram analisados pelo teste $t$ de Student pareado. Houve melhora significativa $(\mathrm{p}<0.01)$ na Melhor Acuidade Visual Corrigida (BCVA) do olho tratado. Inicialmente a média era $72 \pm 8,0$ evoluindo após 40 dias para 75,54 $\pm 6,4$ e depois para 78,27 $\pm 6,9$ após 90 dias (Gráfico 1). Considerando que cada linha tem cinco letras, isso significa que houve uma melhora média da acuidade visual de pouco mais de uma linha (6,27 letras). O poder dióptrico médio (PDM) reduziu em média $0,4 \mathrm{D}$ nos $3 \mathrm{~mm}$ centrais (de 46,67 $\pm 1,9 \mathrm{D}$ para 46,27 $\pm 1,9 \mathrm{D}$, com $\mathrm{p}<0,01$ ) e $0,33 \mathrm{D}$ nos $5 \mathrm{~mm}$ centrais (de 45,04 $\pm 1,5 \mathrm{D}$ para $44,71 \pm 1,6 \mathrm{D}$, com $\mathrm{p}<0,01)$ após 90 dias. Trinta e um pacientes $(83,8 \%)$ evoluíram com diminuição de até $1,3 \mathrm{D}$ no PDM dos 3 milímetros centrais
(Figura 3) e seis pacientes (16,2\%) evoluíram com aumento de até $0,3 \mathrm{D}$. O valor ceratométrico máximo nos $3 \mathrm{~mm}$ centrais reduziu em média $0,6 \mathrm{D}$ (de 54,00 $\pm 3,7 \mathrm{D}$ para 53,39 $\pm 3,8 \mathrm{D}$, com $\mathrm{p}<0,01)$ após 90 dias.

\section{Discussão}

Embora o intervalo de 90 dias seja insuficiente para avaliarmos a estabilização da doença, os trabalhos realizados em populações de outros países ${ }^{(11,13)}$ demonstram que é neste período ( 3 meses) onde ocorrem as principais alterações dos parâmetros ceratométricos e de acuidade visual atribuídos a esta técnica (CXL). Em contraste com os resultados publicados no exterior ${ }^{(11,13)}$, encontramos uma regressão menos acentuada do valor ceratométrico máximo $(0,6 \mathrm{D})$ e do poder dióptrico médio da córnea $(3 \mathrm{~mm} \rightarrow 0,4 \mathrm{D} ; 5 \mathrm{~mm} \rightarrow 0,33 \mathrm{D})$ após três meses de seguimento. A maioria dos pacientes $(83,8 \%)$ evoluiu com melhora dos parâmetros ceratométricos nos $3 \mathrm{~mm}$ centrais, os demais $(16,2 \%)$ apresentaram um aumento máximo de apenas $0,3 \mathrm{D}$, o que não deve ser entendido necessariamente como piora, considerando-se que a reprodutibilidade do exame no aparelho Orbscan é de aproximadamente $0,25 \mathrm{D}$. A melhora da acuidade visual $(6,27$ letras $)$ foi significativa $(\mathrm{p}<0,01)$ e aferida utilizando tabelas padronizadas no modelo ETDRS e com iluminação da sala controlada. Embora haja pequena melhora dos índices topográficos e da acuidade visual, a reticulação do colágeno corneano não deve ser considerada um procedimento refrativo. A indução da reticulação do colágeno é um procedimento de baixo custo, que pode ser muito útil principalmente em regiões onde há pouca disponibilidade de tecido corneano para a realização de transplantes. O efeito de enrijecimento do tecido corneano já foi comprovado in vitro ${ }^{(12)}$, mas a técnica (CXL) ainda necessita de um maior número de estudos para melhor quantificar sua influência sobre os índices topográficos e de acuidade visual nos pacientes com ceratocone.

\section{ConClusão}

Observamos uma melhora menos acentuada dos índices topográficos e de acuidade visual em comparação aos resultados encontrados em outras populações. São necessários mais estudos, com um maior número de pacientes, e maior tempo de seguimento, para que esta técnica confirme seu propósito de estabilização da doença com apenas uma aplicação, e possa talvez se tornar, no futuro, o método de escolha para o tratamento do ceratocone. 


\section{Agradecimentos:}

Os autores são gratos a todos que de alguma forma contribuíram para a realização deste trabalho, e em especial:

- Ao Sr. Fábio Caribé da empresa Visional ${ }^{\circledR}$, por acreditar em nosso projeto e ceder sem qualquer ônus o aparelho Vega CBM X-linker ${ }^{\circledR}$.

- À empresa Allergan ${ }^{\circledR}$, bem representada pelos funcionários Sheila Dias e Hélio Paulo, sempre dispostos a contribuir com nossas pesquisas, e responsáveis pelo fornecimento das amostras de colírio utilizados.

- Ao Dr. Acácio Alves de Souza Lima Filho, da empresa Ophthalmos ${ }^{\circledR}$, que aceitou o desafio de elaborar a solução de riboflavina para crosslinking e nos enviou os frascos sem qualquer custo.

- À diretoria do Hospital de Olhos Niterói, por permitir a realização gratuita de exames no aparelho Orbscan $^{\circledR}$ existente em suas instalações, e às prestimosas funcionárias Danielle Amaral Cruz e Ana Priscila Secundino Peixoto, que colaboraram no agendamento e atendimento dos pacientes.

- Às assessoras de imprensa do HUCFF ( $\mathrm{Sr}^{\mathrm{a}}$.Geiza Porto) e da SBO ( $\mathrm{Sr}^{\mathrm{a}}$. Eleonora Monteiro), pela divulgação do nosso projeto.

\section{Abstract}

Purpose: Assess the clinical and topographical effects of corneal collagen crosslinking in patients with keratoconus on Brazilian population. Methods: Thirty seven eyes of 37 patients (22 female and 15 male) with diagnosis of progressive keratoconus and corneal paquimetry higher than 395 micra, were treated with riboflavin and ultraviolet A radiation, over 30 minutes, to induce corneal collagen crosslinking. Keratometric values were compared as well as the best corrected visual acuity, before and after the procedure, with ranges of 40 and 90 days. Results: There was a reduction of mean dioptric power (PDM) in central $3 \mathrm{~mm}$ in $83.8 \%$ of patients after three months. The PDM reduced $0.4 D$ on average in central $3 \mathrm{~mm}(p<0,01)$ and $0.33 \mathrm{D}$ in central $5 \mathrm{~mm}(p<0,01)$. The maximum keratometric value decreased $0.6 D$ on average $(p<0,01)$. The corrected visual acuity of the treated eye evolved with improvement average of 6,27 letters $(p<0,01)$ after 90 days. Conclusion: We could observe a less pronounced improvement of the topographical values and visual acuity in comparison of the findings in other populations. Further studies with a larger number of patients and longer follow-up are necessary to confirm this technique's purpose of stabilizing the disease with only one application, and allow that to become the method of choice for keratoconus treatment.

Keywords: Keratoconus/drug therapy; Keratoconus/ metabolism; Collagen/radiation effects; Photosensitizing agents/therapeutic use; Riboflavin/therapeutic use; Ultraviolet rays; Corneal topography

\section{REFERÊNCIAS}

1. Mano EB, Mendes LC. Introdução a polímeros. 2a ed. São Paulo: Blücher; 1999.

2. Andrade CT, Coutinho FMB, Dias ML, Lucas EF, Oliveira CMF, Tabak D. Dicionário de polímeros. Rio de Janeiro: Interciência; 2001.

3. Prockop DJ, Kivirikko KI, Tuderman L, Guzman NA. The biosynthesis of collagen and its disorders (first of two parts). N Engl J Med. 1979;301(1):13-23.

4. Yamauchi M, Woodley DT, Mechanic GL. Aging and crosslinking of skin collagen. Biochem Biophys Res Commun. 1988;152(2):898-903.

5. Costa HM, Visconte LLY, Nunes RCR, Furtado CRG. Aspectos históricos da vulcanização. Polímeros. 2003; 13(2):125-9.

6. Carpentier A. From valvular xenograft to valvular bioprosthesis (1965-1977). Med Instrum. 1977;11(2):98-101.

7. Harner $\mathrm{CH}, \mathrm{McC}$ arey BE, Rao PR, Chow AA. In vivo evaluation of biomaterials for synthetic epikeratoplasty [abstract]. Invest. Ophthalmol. Vis. Sci. 1996;37: S68.

8. Leopold LU, Jan CA, Albert DG, inventors. Photochemical cross-linking of polymers [Internet]. United States patent US 3278305. 1966. Available from: www.freepatentsonline.com/ 3278305.html

9. Spoerl E, Huhle M, Seiler T. Induction of cross-links in corneal tissue. Exp Eye Res. 1998;66(1):97-103.

10. Cannon DJ, Foster CS. Collagen crosslinking in keratoconus. Invest Ophthalmol Vis Sci. 1978;17(1):63-5.

11. Wollensak G, Spoerl E, Seiler T. Riboflavin/ultraviolet-ainduced collagen crosslinking for the treatment of keratoconus. Am J Ophthalmol. 2003;135(5):620-7.

12. Wollensak G, Spoerl E, Seiler T. Stress-strain measurements of human and porcine corneas after riboflavin-ultraviolet-Ainduced cross-linking. J Cataract Refract Surg. 2003;29(9):1780-5.

13. Caporossi A, Baiocchi S, Mazzotta C, Traversi C, Caporossi T. Parasurgical therapy for keratoconus by riboflavin-ultraviolet type A rays induced cross-linking of corneal collagen: preliminary refractive results in an Italian study. J Cataract Refract Surg. 2006;32(5):837-45.

\author{
ENDEREÇO PARA CORRESPONDÊNCIA \\ Ricardo Lamy \\ Hospital Universitário Clementino Fraga Filho \\ Av. Brigadeiro Trompowsky s/n $\mathrm{n}^{\mathrm{o}}-\mathbf{1 1}^{\circ}$ andar \\ Dept. de Otorrino e Oftalmologia - Bloco F - Sala 14 \\ CEP 21941-590 - Ilha do Fundão - Cidade Universitá- \\ ria - RJ \\ Telefax: (21) 2590-0846 \\ E-mail: lamy@ufrj.br
}

